\title{
Neonatal mortality in a public referral hospital in southern Haiti: a retrospective cohort study
}

\author{
Alka Dev ${ }^{1,2^{*}} \mathbb{D}$, Michelucia Casseus ${ }^{2,3}$, Wilhermine Jean Baptiste ${ }^{2,3}$, Emma LeWinter ${ }^{2}$, Patrice Joseph ${ }^{4}$ and \\ Peter Wright ${ }^{2}$
}

\begin{abstract}
Background: Haiti has the highest rate of neonatal mortality in the Latin America and Caribbean region. While the rate of facility births in Haiti has doubled over the past two decades, there have been no comparable reductions in maternal or neonatal mortality. Little data is available on the clinical characteristics of complications and morbidities among newborns requiring hospitalization after birth and their contribution to neonatal mortality. There is a need to better understand the status of newborn clinical care capacity in Haiti to prioritize training and resources.

Methods: We performed a retrospective observational cohort study of neonates admitted to a large public referral hospital in southern Haiti in the first 2 years of operation of a new neonatal unit that we established. All neonate cases hospitalized in the unit in these 2 years were reviewed and analyzed to identify their clinical characteristics and outcomes. Multivariable logistic regression was used to identify independent risk factors of hospital mortality. We present the outcomes for 1399 neonates admitted to the unit during August 2017 and August 2019.

Results: The leading cause of death was prematurity, followed by hypoxia and infection. Inborn neonates had better rates of hospital survival than those born elsewhere; they were also more likely to be born via cesarean section and to be admitted immediately following birth. There were no differences between the proportion of premature or lowbirth-weight babies born at the hospital or elsewhere. Mortality in the second year of the unit's operation was 12\%, almost half that of the first year (21\%). Multivariable regression analysis showed that mortality was consistently higher among premature and very low birthweight babies.

Conclusions: With modest investments, we were able to halve the mortality on a neonatal unit in Haiti. Resources are needed to address prematurity as an important outcome since hospital mortality was significant in this group. To this end, investment in uninterrupted supplies of oxygen and antibiotics, as well as ensuring adequate newborn resuscitation, infection control, laboratory testing, and timely morbidity and mortality reviews would go a long way toward lowering hospital mortality in Haiti.
\end{abstract}

Keywords: Neonatal mortality, Prematurity, Hypoxia, Sepsis, Haiti, Newborn care, Neonatal morbidity, Hospital mortality

*Correspondence: alka.dev@dartmouth.edu

2 Dartmouth-Hitchcock Medical Center, Lebanon, NH, USA

Full list of author information is available at the end of the article

\section{Background}

Haiti has struggled with multiple development setbacks over the past few decades, impeding its capacity to respond effectively to the healthcare needs of its people [1]. The country's neonatal mortality rate, estimated 
to be 25 per 1000 live births, is the highest in the Latin America and Caribbean region compared to the regional rate of 9 per 1000 births [2]. Of the 16,427 Haitian infants who died before their first birthday in 2019, 60\% died within the first 28 days of life, the neonatal period; $70 \%$ of these neonatal deaths occurred in the first week [3]. With an increase in facility births in low-income countries, survival for neonates is generally expected to improve. In a systematic review of the effects of healthfacility delivery on neonatal mortality, Tura and colleagues showed that neonatal mortality was $29 \%$ lower in facility births than home births [4]. While the rate of facility births in Haiti has doubled to $40 \%$ over the past two decades, this has not led to comparable reductions in maternal or neonatal mortality and hospital neonatal mortality in Haiti remains high [5-7]. Further, women report disrespect, maltreatment, and isolation at health facilities, impacting their decision to seek medical care and preference for delivering at home $[8,9]$. The national health budget declined four-fold between 2004 and 2016, with greater reliance on international assistance and outof-pocket payments even though poverty is a severe barrier to accessing medical care [10]. Thus, maintenance of even the most basic public-health functions has been challenging given the current financing and governance structures. Understandably, Haiti lags far behind other countries in achieving its public health targets.

Haiti has a three-tiered health care system designed to improve primary care access at the community level [1113]. Maternal and newborn health services are overseen by the Department of Family Health within the Ministry of Public Health and Population to ensure that every birth is attended by a qualified provider. Tier 1, primary care, has 3 levels. The first level includes dispensaries that provide essential medicines and are staffed by community health workers - screening for risk factors during pregnancy and newborn vaccinations are available here. Level 2 includes Health Centers (with or without beds) that are staffed by medical personnel who provide basic primary care services, screening for sickle cell anemia, management of risk factors, HIV testing and treatment, low- to moderate-risk vaginal deliveries, and follow-up of sick newborns are available here. Level 3 includes Community Reference Hospitals offering basic inpatient clinical services - high-risk vaginal deliveries are done here. Tier 2, secondary care, includes Departmental Hospitals which serve as the reference institution for each of the 10 Departments - they can provide cesarean sections and management of newborn referral cases from the lower levels. Tier 3, tertiary care, includes academic hospitals and specialized national institutions - their role in maternal and newborn care is to provide specialty services but Haitian women also seek care here for normal deliveries. While $91 \%$ of Haitians live within $5 \mathrm{~km}$ of a primary care facility, most of these facilities are cited for inefficiency and low-quality care due to low productivity, absenteeism, and stockouts [14]. Vast disparities remain between urban and rural facilities in terms of obstetric service delivery readiness and newborn care $[15,16]$.

The lack of appropriate prenatal care, the low quality of obstetric care, and high rates of eclampsia, obstructed labor, untreated infection, and preterm delivery are persistent factors behind the high rates of maternal and neonatal mortality in Haiti $[6,7,17,18]$. In a recent analysis, we reported that $2-5 \%$ of deliveries in hospitals in southern Haiti suffered from eclampsia, which was likely an underestimation due to poor diagnostic capacity and data quality [19]; an eclampsia rate of $23 \%$ was reported in another Haitian hospital with better electronic recordkeeping [20]. Lack of medical supervision, essential newborn care services, and an ability to respond to emergent situations during birth can contribute to birth asphyxia, infection, and prematurity-related complications, which are major causes of neonatal mortality in low-income countries [21]. Rural hospitals in Haiti often lack the capacity to care for newborns after birth due to gaps in essential infrastructure, equipment, supplies, medicines, and staff trained to implement up-to-date protocols and guidelines [16]. In addition to improving skilled care at birth, we need to generate evidence regarding the provision of skilled care to neonates requiring hospitalization. Case management in neonatal care units can inform service delivery and quality improvement initiatives for newborn morbidity, mortality, and survival [22].

To care for newborns requiring hospitalization in Haiti, we worked with the leadership at Hopital Immaculae Conception (HIC), a departmental hospital in Sud, to establish a neonatal care unit for newborns needing medical care after birth. The objective of this study was to conduct a retrospective review of neonatal admissions during the first 2 years of the unit's operation to identify risk factors for hospital neonatal mortality, determine if survival improved over time, and inform newborn care based on our analysis.

\section{Methods \\ Study setting}

HIC is the main departmental referral hospital (secondary care), located in Les Cayes and serving a population of 774,976 (Fig. 1). There are 18 communes (or subdepartmental administrative units) in Sud, of which Les Cayes is the largest with an approximate population of 140,327 people [23]. The maternity ward has an annual volume of 3000-4000 deliveries [19]. At the time of our project, the maternity ward had two beds in the delivery room and was staffed by a midwife or a nurse who 


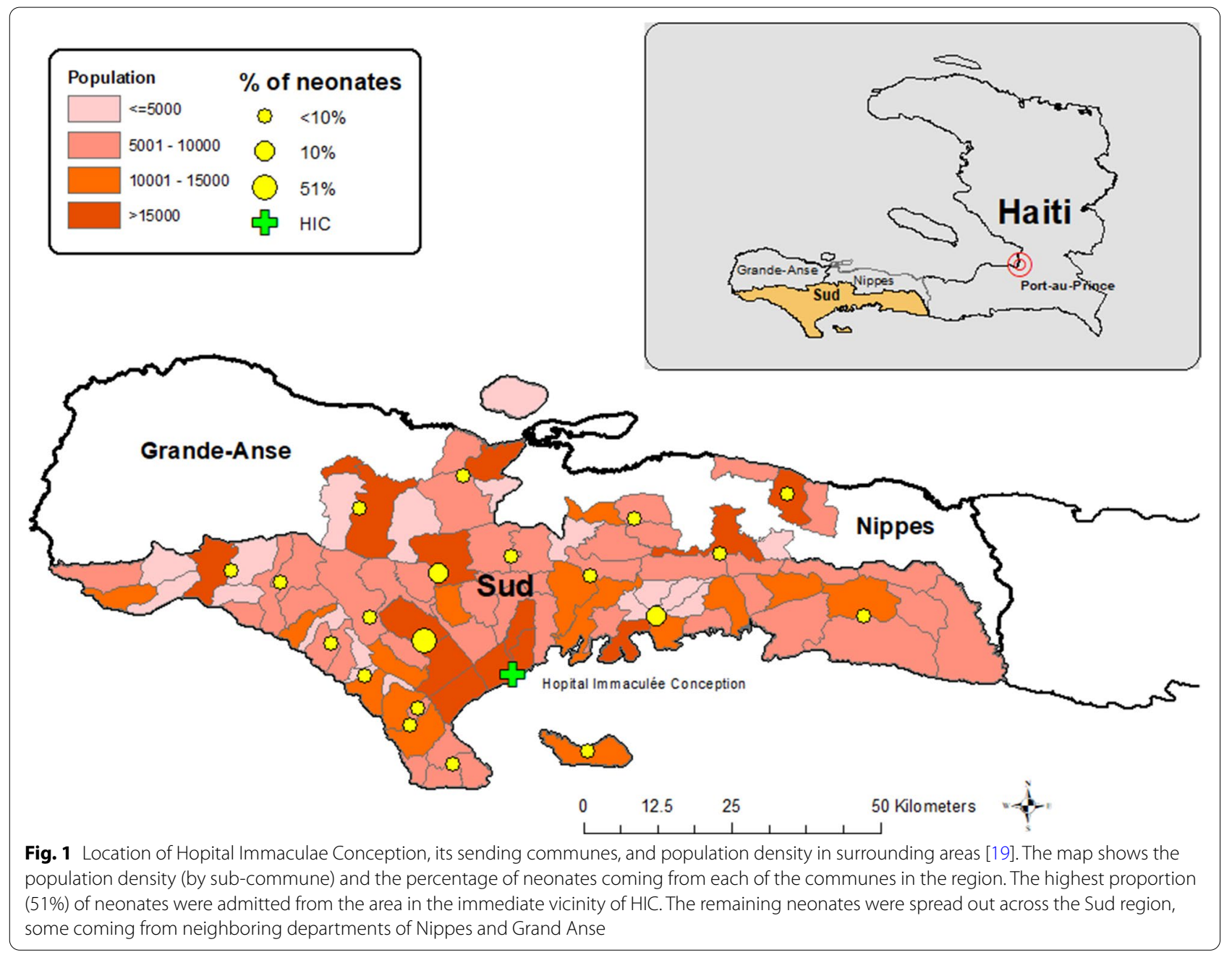

attended deliveries and provided newborn resuscitation, as needed. Obstetricians attended some of the more complicated deliveries and performed cesarian sections. A warmer was available but never used as the staff was under the assumption that it did not work because a message showing it was warming up would appear in red and staff assumed it was an error message and turned it off. We corrected this misunderstanding but did not document if the warmer was used consistently afterward. Normal procedures following a low-risk birth included weighing the baby, giving vitamin $\mathrm{K}$, and applying ophthalmic tetracycline ointment. There was no oxygen in the delivery room, but suction and/or resuscitation with a bag valve mask was performed as needed. If a baby needed oxygen, an oxygen concentrator was used if there was electricity. No intubation was ever performed on the delivery ward. Babies in need of oxygen or intubation were referred out and likely did not survive the journey. Water and soap were available, but hand sanitizer use was more common.
Most women arrived at the maternity ward shortly before birth and after laboring at home. Accurate estimates of gestational age were generally not available. Clinical assessment and the date of the last menstrual period were used to establish the gestational age to determine prematurity at the time of delivery. Approximately $15 \%$ of births were through cesarean section [19]. Breastfeeding was generally not initiated immediately after birth or promoted in the maternity ward. There was no specific staff assigned for newborn care nor adequate space, equipment, or material for case management. The pediatric unit was located next to the maternity ward and received newborns, but those in need of critical care were referred to a hospital that was more than $2 \mathrm{~h}$ away.

\section{Neonatal unit structure and function}

In June 2017, a team from Dartmouth and GHESKIO, with funding from the Children's Prize and the WK Kellogg Foundation, established the first neonatal care unit at HIC. The unit functioned at a Level IIA neonatal 
capacity, caring primarily for newborns who were ill with problems that were expected to resolve quickly with moderate risk of serious complications and no assisted ventilation [24]. Two pediatricians were appointed to provide care on an 8-bed unit, which was later expanded to 13 beds due to high demand. The hospital administration hired 8 nurses to staff the unit. Other support staff included a project coordinator, a data manager, a lab coordinator, three community health workers, and four cleaners. All staff was supported through project funding. Pediatricians alternated each week on inpatient and outpatient follow-up services. One pediatrician was on call every night and weekend. A third pediatrician provided vacation coverage as needed.

Renovation and expansion of the space, as well as provision of essential equipment, medications, oxygen, and supplies, were completed sequentially over 2 years. We note that there were two incubators in use but due to inconsistent power supply, these were not always reliable. Additional equipment purchased and provided through our grant included eight warmer-bassinets, three oxygen concentrators, four incubators, and a portable ultrasound (for maternity ward). Toward the end of our project, electricity was regularly supplied on the neonatal ward due to the installation of a new generator at the hospital. There were no ventilators on the ward. Although nursing and administrative staff were later integrated into the hospital's operating budget, the initial investment and technical oversight were provided by our team. All staff reported to supervisors within the hospital and were integrated into the hospital's human resource structure.

\section{Staff training and services}

The two pediatricians (MC and WJB) received 3 months of supervised neonatal training at Dartmouth-Hitchcock Medical Center in the U.S., and GHESKIO and St. Boniface Hospital in Haiti. This included a master training in Helping Babies Breathe (HBB) at Dartmouth [25]. There was no postgraduate training available for neonatal care at HIC and no pediatric residents rotated through the hospital as it was not an academic hospital. Neonatal training for nurses was delivered at hiring and after 18 months of service by visiting neonatal nurses from the U.S. and Haiti. In the interim, a visiting Dartmouth pediatric resident provided on-the-job training to nurses in HBB and Essential Care for Every Baby [26] curricula, jaundice care, shift handoff, head-to-toe exams, intake assessments, and Kangaroo Mother Care (KMC). We also reviewed nursing charts and redesigned nursing forms to ease shift handoff, sign off on doctors' orders, reduce redundant note-writing, and properly document medications. Nurses encouraged all mothers and fathers to provide KMC. Breastfeeding was encouraged and promoted when a neonate was admitted and if the mother was able to initiate. Manual breast pumps were provided to mothers in recovery after a cesarean or for neonates who were unable to latch on. The pediatricians and nurses made every effort to promote breastfeeding, but the lack of comfortable seating remained a barrier.

\section{Data sources and outcomes}

Patients were admitted to the neonatal care unit from the maternity ward at HIC or were born at other facilities/homes in the region and transferred to the unit by their families. In a small number of cases, newborns were transferred by ambulance from referring facilities. The admitting staff included both pediatricians and nurses. A daily electronic register documenting the date of birth, date of admission, place of birth, weight at admission, prematurity status, major health issue(s), length of stay, and outcome for all infants admitted to the unit, was maintained by the pediatricians in MS Excel@. Data were reviewed quarterly by the pediatricians and DartmouthGHESKIO teams to track progress, review mortality cases, and identify major bottlenecks. The final database for this study was a compilation of all admissions to the unit between August 2017 and August 2019. The outcome of interest was an inpatient neonatal death.

\section{Diagnostic criteria}

Based on the assessment of the pediatricians, primary diagnoses were established based on the International Classification of Diseases and Related Health Problems (ICD-10) maintained by the WHO [27]. Due to limited laboratory and diagnostic resources, the pediatricians were unable to confirm their diagnoses with more than minimal blood work. Microbiologic definitions, other than HIV and syphilis screening tests, were not available. Prematurity was defined as gestational age less than 37 weeks, which was established using the Ballard Score Maturational Assessment of Gestational Age in Newly Born Infants [28]. Low birth weight (LBW) at admission was defined as weight less than $2500 \mathrm{~g}$ and very low birth weight (VLBW) as less than $1500 \mathrm{~g}$. A perinatal hypoxia diagnosis was given to babies that had "no cry at birth," meconium aspiration, surfactant deficiency, fetal distress, and/or secondary hypoxic injury, such as Hypoxic Ischemic Encephalopathy (HIE). Acute respiratory distress syndrome was reserved for preterm newborns with severe illness, such as sepsis, pancreatitis, or pneumonia, in addition to hypoxia. HIE was diagnosed a few hours after birth by using Sarnat classification and the birth history context [29]. Jaundice was diagnosed based on clinical signs such as the color of the skin and mucosa, and grade by localization of the yellow coloration in the body or mucosa and level of blood Bilirubin. Treatment 
for higher-than-normal levels of Bilirubin was phototherapy and sometimes, phenobarbital. Hypoglycemia was diagnosed using a dipstick test of blood sugar at admission and clinical signs like lethargy and poor suction. Treatment was administered in the form of IV Dextrose 10: $2 \mathrm{cc} / \mathrm{kg}$ stat. Another test was administered after $15 \mathrm{~min}$ and if positive, another bolus of Dextrose 10 was administered.

\section{Exclusion criteria}

A flow chart shows the derivation of the sample and reasons for exclusion (Fig. 2). Very small neonates weighing less than $1 \mathrm{~kg}(n=12)$ were excluded from the dataset due to their exceptionally poor survival prognosis. Infants older than 28 days were also excluded $(n=11)$. All neonates taken home against medical advice or transferred out of the unit were excluded from the multivariable analysis, but we compared their baseline characteristics to identify any patterns among those who left. Parents' reasons for leaving early were numerous: having other children to care for at home; timing with major festivals; lack/cost of room and board for families; and political unrest and insecurity. We suspected that some parents also wanted the child to receive traditional medicine at home or were not confident that the baby could recover at the hospital.

\section{Analyses}

Neonates who were born at HIC were compared to those who were born elsewhere (at home or another facility) to identify any observable differences in neonatal characteristics using a Pearson's chi-squared test for categorical variables and Student's t-test for mean length of stay. (Table 1). Next, we tested for bivariate relationships between those who died and those who were discharged, excluding neonates whose parents took them home early against medical advice, to identify significant factors contributing to mortality in the neonatal unit (Table 2). Multivariable analysis was performed on the same dataset to calculate adjusted odds ratios of dying on the ward.

\section{Patient and public involvement}

As this study was based on retrospective data review, patients were not involved in choosing the methods or agreeing to plans for dissemination of the study results to parents and their communities.

\section{Results}

\section{Neonatal characteristics}

Half of the patients admitted to the neonatal unit at HIC were born to mothers from Les Cayes, the commune in which HIC is located (Fig. 1). Another 10\% of babies were born to moms in the nearby Torbeck commune which has a birthing center with ambulance availability. The remaining cases came from the rest of Sud and communes in departments sharing a border with Sud. We did not ascertain why some of the neonates came from mothers in other departments; possibly they stayed with family living near HIC.

Table 1 presents the characteristics of all neonates admitted to the unit between August 2017 and August 2019 by place of birth. In all, 1399 neonates who were 28 days of age or younger and weighed more than $1 \mathrm{~kg}$ at admission were admitted to the unit; $66 \%$ were "inborn," meaning born at HIC $(n=922)$, and the rest were "outborn," meaning born at home or another facility $(n=477)$. There were very few differences in the recorded baseline characteristics of patients by place of birth. The proportions of boy versus girl neonates were similar for those born at HIC and elsewhere. Preterm birth complicated one-fifth of all admissions regardless of the place of

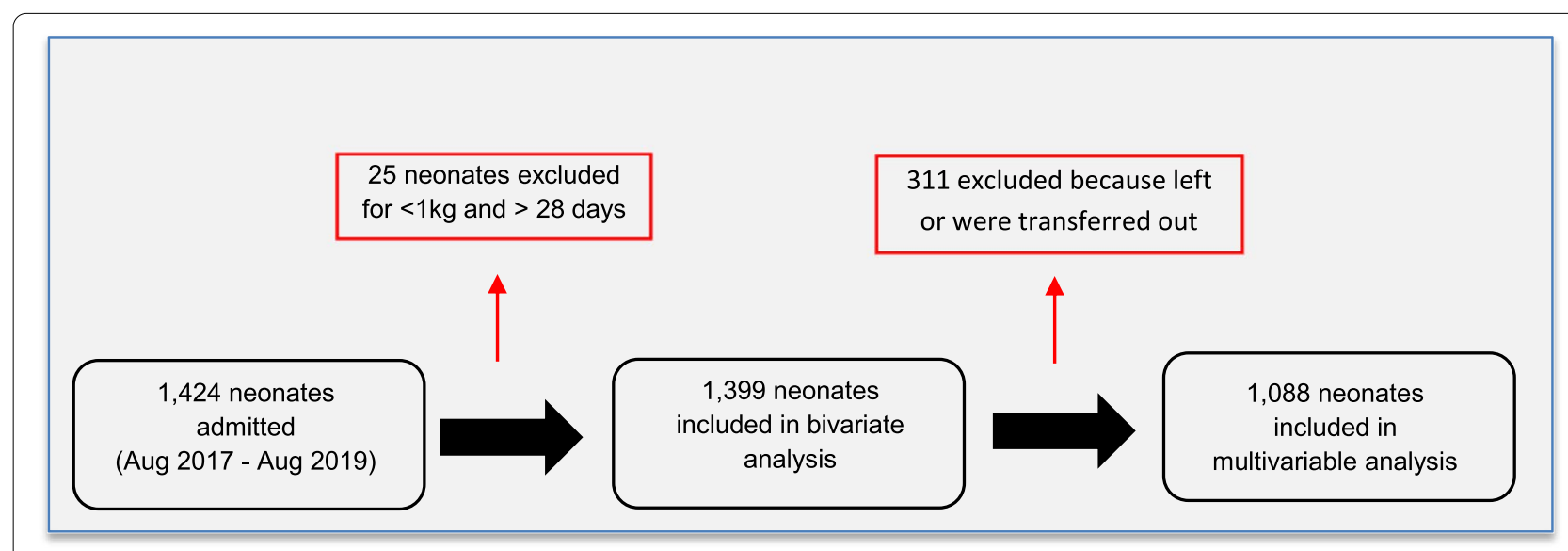

Fig. 2 Sample calculation 
Table 1 Baseline characteristics of 1399 neonatal inpatients at Hopital Immaculae Conception, Les Cayes by place of birth (2017-2019)

\begin{tabular}{|c|c|c|c|c|}
\hline & Outborn & Inborn & Total & $p$-value \\
\hline$N(\%)$ & $477(34.1)$ & $922(65.9)$ & $1399(100.0)$ & \\
\hline Sex & & & & 0.164 \\
\hline Male & $288(60.4)$ & $521(56.5)$ & $809(57.8)$ & \\
\hline Female & $189(39.6)$ & $401(43.5)$ & $590(42.2)$ & \\
\hline Age at admission & & & & 0.000 \\
\hline Day of birth & $128(26.8)$ & $578(62.7)$ & $706(50.5)$ & \\
\hline 1-6 days after birth & $219(45.9)$ & $271(29.4)$ & $490(35.0)$ & \\
\hline 7-28 days after birth & $130(27.3)$ & $73(7.9)$ & $203(14.5)$ & \\
\hline Timing of birth & & & & 0.804 \\
\hline Term & $386(80.9)$ & $741(80.4)$ & $1127(80.6)$ & \\
\hline Preterm & $91(19.1)$ & 181 (19.6) & $272(19.4)$ & \\
\hline Outcome & & & & 0.000 \\
\hline Died & $69(14.5)$ & $102(11.1)$ & $171(12.2)$ & \\
\hline Discharged & $274(57.4)$ & $643(69.7)$ & $917(65.5)$ & \\
\hline Left against Dr's orders & $123(25.8)$ & $168(18.2)$ & $291(20.8)$ & \\
\hline Transferred & $11(2.3)$ & $9(1.0)$ & $20(1.4)$ & \\
\hline Multiple gestations & & & & 0.472 \\
\hline Singleton & $435(91.2)$ & $851(92.3)$ & $1286(91.9)$ & \\
\hline Twin or more & $42(8.8)$ & $71(7.7)$ & $113(8.1)$ & \\
\hline Birthweight categories & & & & 0.233 \\
\hline $1.0-1.4 \mathrm{~kg}$ & $35(7.3)$ & $52(5.6)$ & $87(6.2)$ & \\
\hline $1.5-2.4 \mathrm{~kg}$ & $145(30.4)$ & $258(28.0)$ & $403(28.8)$ & \\
\hline $2.5+\mathrm{kg}$ & $297(62.3)$ & $612(66.4)$ & $909(65.0)$ & \\
\hline Type of Delivery & & & & 0.000 \\
\hline Vaginal & $436(91.4)$ & $690(74.8)$ & $1126(80.5)$ & \\
\hline Cesarean & $41(8.6)$ & $232(25.2)$ & $273(19.5)$ & \\
\hline Age of mother & & & & 0.172 \\
\hline$<18$ years & $17(3.6)$ & $45(4.9)$ & $62(4.4)$ & \\
\hline $18-35$ years & $368(77.1)$ & 737 (79.9) & 1105 (79.0) & \\
\hline$>35$ years & 76 (15.9) & $112(12.1)$ & $188(13.4)$ & \\
\hline Unknown & $16(3.4)$ & $28(3.0)$ & $44(3.1)$ & \\
\hline $\begin{array}{l}\text { Mean number of living } \\
\text { children }\end{array}$ & 1.3 & 0.98 & 1.09 & 0.0001 \\
\hline $\begin{array}{l}\text { Mean length of stay } \\
\text { (days) }\end{array}$ & 4.3 & 4.3 & 4.3 & 0.977 \\
\hline
\end{tabular}

Table 2 Case fatality by clinical diagnosis ${ }^{\mathrm{a}}$

\begin{tabular}{llll}
\hline Diagnosis & $\begin{array}{l}\text { Number } \\
\text { admitted }\end{array}$ & Case fatality (\%) & $\begin{array}{l}\text { Proportion of } \\
\text { all deaths (\%) }\end{array}$ \\
\hline Prematurity & 182 & $61(33.5 \%)$ & $35.7 \%$ \\
Hypoxia & 215 & $26(12.1 \%)$ & $15.2 \%$ \\
Suspected Infection & 396 & $23(5.8 \%)$ & $13.4 \%$ \\
Hypoxia + Infection & 113 & $13(11.5 \%)$ & $7.6 \%$ \\
Encephalopathy & 71 & $26(36.6 \%)$ & $15.2 \%$ \\
Other & 111 & $22(19.8 \%)$ & $12.9 \%$ \\
Total & 1088 & $171(15.7 \%)$ & $100 \%$ \\
\hline
\end{tabular}

${ }^{a}$ Does not include those who left without discharge birth. Less than 1 in 10 babies were from multiple gestation pregnancies. Differences in the neonates' weights on admission and mothers' ages were also insignificant by place of birth. Close to one-third of neonates were low or very low birth weight, regardless of the place of birth. A majority of mothers (79\%) were between 18 and 34 years of age and $62(4.4 \%)$ were 18 or under.

The three factors that were significantly different by place of birth were: survival status, the mode of delivery, and age at admission. Mortality was higher among outborn babies (14.5\% versus $11.1 \%$ among babies born at $\mathrm{HIC}$ ) and outborn babies were also more likely to be taken home against medical advice $(25.8 \%$ versus $18.2 \%$ among babies born at HIC). The mode of delivery was vaginal for most births, but more so for outborn babies, which included home births (91.4\% among outborn versus $74.8 \%$ among inborn). The majority of outborn babies were brought in within 1-6 days of birth (45.9\%), compared to those born at HIC where the majority were transferred on the day of birth (62.7\%). However, most of the admissions were made in the first week of life for all neonates. A higher proportion of births at HIC were through cesarean section (25\%) compared to those born elsewhere (9\%). Both sets of neonates had similar lengths of hospital stay (mean $=4.3$ days).

Triage at birth to identify babies at risk was not consistently followed on the maternity ward as $17.3 \%$ of babies born at HIC were only admitted to the neonatal unit a day after birth. These babies were either kept in the maternity ward with their mother, where they deteriorated, or sent home and brought back by family members because of illness. Although we do not show the data, home births were more likely to be admitted later than births from other facilities.

\section{Characteristics of babies taken home against doctor's orders}

To analyze survival until discharge, we excluded babies who were taken home early by their parents and only included those who either died during their stay or were discharged by the attending pediatrician. Chi-square comparisons of included and excluded babies showed that there were no significant differences between those taken home early by parents versus those staying until discharge or death. Regarding birth weight, babies who were taken home early matched proportionally to those who remained until discharge or death $\left(\mathrm{X}_{3}^{2}=0.45\right.$, $p=0.930)$. There were no differences in the diagnoses of prematurity $\left(\mathrm{X}^{2}{ }_{1}=0.86, p=0.353\right)$, infection $\left(\mathrm{X}^{2}{ }_{1}=0.03\right.$, $p=0.865)$, hypoxia $\left(\mathrm{X}^{2}{ }_{1}=1.53, p=0.215\right)$, or encephalopathy $\left(\mathrm{X}^{2}{ }_{1}=1.04, p=0.307\right)$ among the two groups. However, Student's t-test showed that babies taken home early by their parents went home a day earlier on 
average (mean $=3.4$ days) compared to those who stayed (mean $=4.5$ days, $\mathrm{t}=4.03, p=0.0001$ ). Mothers who left early with their babies had a higher number of live children at home $($ mean $=1.3)$ compared to those who stayed $($ mean $=1.0, \mathrm{t}=-2.41, p=.0001)$.

\section{Clinical characteristics}

Diagnostic capacity was limited by the lack of confirmatory testing and information on maternal risk factors. The most common health issues experienced by the neonates included suspected infection, hypoxia, and prematurity; neonates often suffered from more than one complication (Fig. 3). Overall, 36\% of neonates were suspected of having an infection, which was treated empirically with ampicillin and gentamycin and/or cefotaxime; $11 \%$ also shared a diagnosis of hypoxia in addition to the suspected infection. One in three neonates suffered hypoxia, with $11 \%$ also having complications from infection as noted above, and $6 \%$ with hypoxia and other complications (not shown separately). Nearly all premature infants were diagnosed as being hypoxic. Other health issues included: congenital malformations; obstetrical complications; jaundice; infections such as tetanus and meningitis; other pulmonary complications; seizures; cardiac conditions; and inappropriate feeding at home.
Encephalopathy was clinically diagnosed in 6\% of cases and included babies with Hypoxic Ischemic Encephalopathy and Hyperbilirubinemia Encephalopathy.

In all, 171 babies (15.7\%) died during their stay at HIC (Table 2). In terms of case fatality, both prematurity and encephalopathy were quite fatal; in both cases, at least a third of infants with these diagnoses died. Another third of deaths occurred in hypoxic babies, suspected of having an infection or diagnosed with both hypoxia and infection. Other diagnoses included meningitis, congenital abnormalities, jaundice, tetanus, intestinal occlusion, and congenital syphilis.

\section{Hospital neonatal mortality vs. discharge}

Table 3 presents results of chi-square tests of independence for hospital mortality versus discharge. As noted, preterm babies were much more likely to die than term babies $\left(\mathrm{X}_{1}^{2}=110.4, p=0.000\right)$. There were notable differences by weight at admission and place of birth; VLBW and LBW babies were much more likely to die than to be discharged $\left(\mathrm{X}_{2}^{2}=126.0, p=0.000\right)$ and babies born elsewhere and transferred to HIC also had higher mortality $\left(\mathrm{X}_{1}^{2}=7.3, p=0.007\right)$ than inborn babies. Infants born via cesarean had better survival $\left(\mathrm{X}^{2}{ }_{1}=11.1, p=0.001\right)$. We also note that neonates

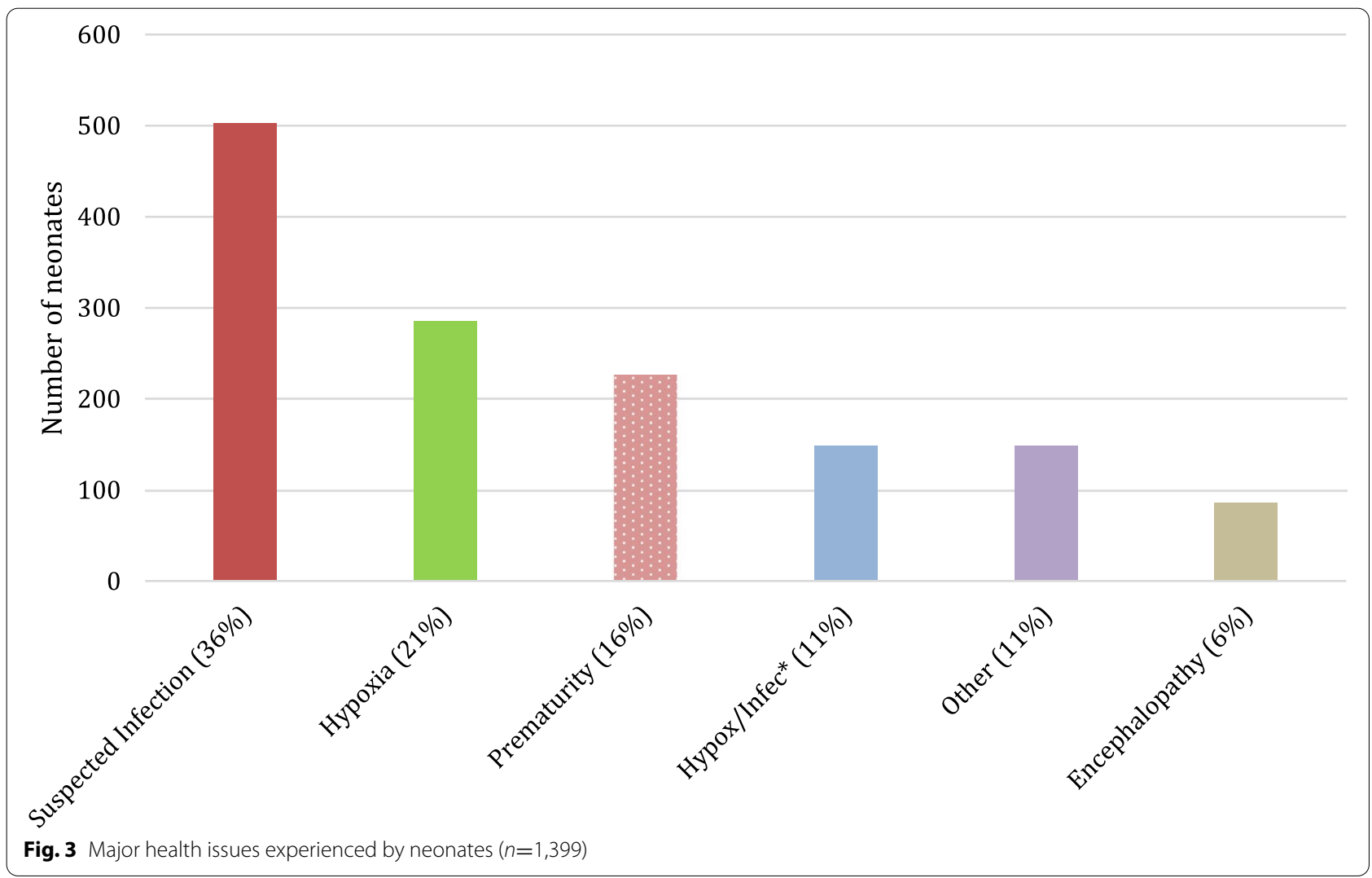


Table 3 Risk factors for mortality among 1088 neonates at Hopital Immaculae Conception, Les Cayes (2017-2019)

\begin{tabular}{|c|c|c|c|c|}
\hline & Discharged & Died & Total & $P$-value \\
\hline$N(\%)$ & $917(84.3)$ & $171(15.7)$ & $1088(100.0)$ & \\
\hline Timing of birth & & & & 0.000 \\
\hline Term & $783(90.1)$ & $86(9.9)$ & $869(100.0)$ & \\
\hline Preterm & $134(61.2)$ & $85(38.8)$ & $219(100.0)$ & \\
\hline Birthweight & & & & 0.000 \\
\hline $1.0-1.4 \mathrm{~kg}$ & $30(43.5)$ & $39(56.5)$ & $69(100.0)$ & \\
\hline $1.5-2.4 \mathrm{~kg}$ & $244(77.2)$ & $72(22.8)$ & $316(100.0)$ & \\
\hline $2.5+\mathrm{kg}$ & $643(91.5)$ & $60(8.5)$ & $703(100.0)$ & \\
\hline Sex & & & & 0.492 \\
\hline Male & $521(83.6)$ & $102(16.4)$ & $623(100.0)$ & \\
\hline Female & $396(85.2)$ & $69(14.8)$ & $465(100.0)$ & \\
\hline Place of birth & & & & 0.007 \\
\hline Outborn & $274(79.9)$ & $69(20.1)$ & $343(100.0)$ & \\
\hline Inborn & $643(86.3)$ & $102(13.7)$ & $745(100.0)$ & \\
\hline Multiple gestations & & & & 0.230 \\
\hline Singleton & $841(84.7)$ & $152(15.3)$ & $993(100.0)$ & \\
\hline Twin or more & $76(80.0)$ & $19(20.0)$ & $95(100.0)$ & \\
\hline Type of delivery & & & & 0.000 \\
\hline Vaginal & $712(82.4)$ & $152(17.6)$ & $864(100.0)$ & \\
\hline Cesarean & $205(91.5)$ & $19(8.5)$ & $224(100.0)$ & \\
\hline Age of mother & & & & 0.014 \\
\hline$<18$ years & $30(68.2)$ & $14(31.8)$ & $44(100.0)$ & \\
\hline $18-35$ years & $741(85.5)$ & $126(14.5)$ & $867(100.0)$ & \\
\hline$>35$ years & $124(84.9)$ & $22(15.1)$ & $146(100.0)$ & \\
\hline Unknown & $22(71.0)$ & $9(29.0)$ & $31(100.0)$ & \\
\hline Age of neonate at admission & & & & 0.000 \\
\hline Day of birth & $435(78.5)$ & $119(21.5)$ & $554(100.0)$ & \\
\hline 1-6 days after birth & $336(90.6)$ & $35(9.4)$ & $371(100.0)$ & \\
\hline 7-28 days after birth & $146(89.6)$ & $17(10.4)$ & $163(100.0)$ & \\
\hline Mean length of stay (days) & 4.6 & 3.9 & 4.6 & 0.075 \\
\hline Mean number of living children & 1.00 & 1.12 & 1.08 & 0.343 \\
\hline Project timing & & & & 0.000 \\
\hline First year of unit operation & $358(79.0 \%)$ & $95(21.0 \%)$ & $635(100.0 \%)$ & \\
\hline Second year of unit operation & $559(88.0 \%)$ & $76(12.0 \%)$ & $453(100.0 \%)$ & \\
\hline
\end{tabular}

admitted on the day of birth experienced higher mortality rates than those admitted a little later $\left(\mathrm{X}_{2}^{2}=28.4\right.$, $p=0.000$ ). There was also an indication that babies born to teenage mothers under 18 years of age had higher mortality compared to other ages $\left(\mathrm{X}_{3}^{2}=13.7\right.$, $p=0.003$ ). Finally, we compared mortality in the first year of the unit's operation (August 2017 - August 2018) to the second year of the unit's operation (September 2018 - August 2019) and found that mortality on the neonatal unit decreased by $40 \%$, from 1 in 5 babies dying in the first year to 1 in 8 babies dying in the second year $\left(\mathrm{X}^{2}{ }_{1}=16.1, p=0.000\right)$. Other demographic characteristics, such as the neonate's sex or being a twin were not correlated with mortality. Student's-test showed that the difference in mean lengths of hospital stay was not significant by survival status but trending toward a somewhat longer stay for those who were discharged ( $\mathrm{t}=1.8, p=0.075)$ and the number of live children was not a risk factor for hospital mortality.

Logistic regression models were supportive of our bivariate findings (Table 4). After adjusting for birth weight and other risk factors, preterm neonates had over twice the odds of dying compared to term babies $(\mathrm{AOR}=2.38 ; p=0.002)$. As expected, LBW and VLBW babies had higher odds of death when compared to normal-weight babies (NBW); LBW babies were 1.7 times 
Table 4 Odds of death in the hospital compared to discharge by risk categories

\begin{tabular}{|c|c|c|c|}
\hline Died & $\begin{array}{l}\text { Adjusted Odds } \\
\text { Ratio (AOR) }\end{array}$ & $\begin{array}{l}\text { (95\% } \\
\text { confidence } \\
\text { interval) }\end{array}$ & $P$-value \\
\hline \multicolumn{4}{|l|}{ Timing of delivery } \\
\hline Term & reference & & \\
\hline Preterm & 2.38 & $(1.39-4.06)$ & 0.000 \\
\hline \multicolumn{4}{|l|}{ Birthweight } \\
\hline VLBW $1.0-1.4 \mathrm{~kg}$ & 5.50 & $(2.58-11.76)$ & 0.000 \\
\hline LBW $1.5-2.4 \mathrm{~kg}$ & 1.68 & $(1.03-2.75)$ & 0.037 \\
\hline NBW $2.5 \mathrm{~kg}+$ & reference & & \\
\hline \multicolumn{4}{|l|}{ Place of birth } \\
\hline HIC (inborn) & reference & & \\
\hline Other (outborn) & 2.20 & $(1.43-3.37)$ & 0.000 \\
\hline \multicolumn{4}{|l|}{ Type of delivery } \\
\hline Vaginal & reference & & \\
\hline Cesarean & 0.47 & $(0.27-0.82)$ & 0.009 \\
\hline \multicolumn{4}{|l|}{ Age of mother } \\
\hline$<18$ years & 3.05 & $(1.35-5.86)$ & 0.004 \\
\hline 18-34years & reference & & \\
\hline$>35$ years & 1.31 & $(0.74-2.13)$ & 0.327 \\
\hline Unknown & 2.04 & $(1.08-6.50)$ & 0.129 \\
\hline \multicolumn{4}{|l|}{ Age at admission } \\
\hline Day of birth & reference & & \\
\hline $1-6$ days & 0.44 & $(0.28-0.71)$ & 0.001 \\
\hline 7 days or more & 0.40 & $(0.22-0.75)$ & 0.004 \\
\hline \multicolumn{4}{|l|}{ Project timing } \\
\hline First year of project & reference & & \\
\hline Second year of project & 0.44 & $(0.30,0.64)$ & 0.000 \\
\hline
\end{tabular}

as likely to die before discharge $(p=0.037)$ and VLBW babies were five times as likely to die $(p=0.000)$. The odds of dying were 2.2 times higher for babies referred to the hospital than those born at HIC $(p=0.000)$. Those born through cesarean section had a survival advantage $(\mathrm{AOR}=0.47 ; p=0.009)$. Mothers who were less than 18 years of age had higher odds of losing their neonate when compared to mothers who were 18-34years old $(\mathrm{AOR}=3.05, p=.004)$; the age of the mother was not a factor in mortality otherwise. Babies admitted 1-6 days after birth $(\mathrm{AOR}=0.44 ; p=0.001)$ or more than a week after birth (AOR $=0.40 ; p=0.004)$ had less than half the odds of dying compared to babies admitted on the day of birth. Finally, after adjusting for observed risk factors, we established that the odds of a neonate dying in the second year of the unit's operation was $56 \%$ lower than in the first half of the project $(\mathrm{AOR}=0.44, p=0.000)$.

\section{Discussion}

This study assessed the neonatal outcomes at a newly formed neonatal care unit in southern Haiti and showed overall mortality of $12.2 \%$ during the first 2 years of the unit's operation. This mortality rate is midrange compared to mortality rates that have been reported in similar settings elsewhere, including southern Eritrea (6.5\%), western Ethiopia (8.8\%), north-central Nigeria (13.4\%), western Haiti (14.5\%), south-eastern Nigeria (19.4\%), eastern Ethiopia (20.0\%), Ghana (20.2\%), and southern Ethiopia (25.0\%) [6, 30-36]. If we exclude those who left the hospital early or were transferred out, we calculate a mortality rate of $15.7 \%$ among those with a known hospital outcome. We also had the opportunity to improve neonatal care through clinician training, renovation, provision of essential equipment and medications, and support for operational systems. There were significant improvements in survival to discharge over the 2 years, increasing from $79.0 \%$ in the first year to $88.0 \%$ in the second.

As seen in other low resource settings, mortality was highest among premature neonates; nearly $40 \%$ of all preterm babies died compared to $10 \%$ of term babies. Among premature neonates in Burundi, a similar disparity in inpatient mortality rate was observed; among those less than 32-weeks gestational age, 31\% died compared to $11 \%$ among those who were $32-36$ weeks' gestation $(11 \%)$ [37]. In India, over half of all neonatal deaths recorded in the National Neonatal Perinatal Database were among preterm babies [38]. Other published estimates of premature mortality are alarming as well; close to $50 \%$ of neonates born at 34 weeks of gestation and $70 \%$ of neonates born at $<32$ weeks of gestation die in low-income countries [39, 40]. Specific gestational age was not available in our dataset. Other causes of death were similar as in other settings - with hypoxia and suspected infection leading the next third of all deaths, half of them attributable to infection. Comparatively, at St. Damien Hospital in Haiti, $23 \%$ of all neonates with sepsis died, although the odds of dying were 2.4 times higher for those who were treated empirically rather than culture-confirmed [7]. Sepsis-related neonatal mortality rates of $14.6-36.0 \%$ have been reported in other settings [41]. Thus, our sepsis results are comparable to those in other studies, although none of the cases were culture-confirmed. Our hypoxiarelated death rates were somewhat lower than other settings $-15.2 \%$ for hypoxia and another $7.6 \%$ for hypoxia complicated by sepsis. Similar rates have been reported for asphyxia-related hospital mortality in Nigeria (24.1\%) and Benin (20.0\%) [33, 42]. Community-level rates of asphyxia-related deaths may be higher - as reported in Nepal (30.0\%) [43]. 
Survival was better among neonates born at HIC, likely due to the higher risks associated with home births or births at facilities without cesarean section or other emergency obstetric services. Lower-level facilities in Haiti are poorly equipped to provide obstetric care, even if they manage labor and delivery [44]. We also found that the risk of dying was highest among babies admitted immediately after birth from HIC although we did not have sufficient data to determine why this may be the case. It is possible these neonates barely survived birth. Generally, mortality rates in favor of inborn babies have been well documented [45]. While the standard practice is to separate community transfers or outborn babies from inborn babies due to the risk of infection, we could not to do so which may have increased the risk of transmission from outborn to inborn neonates [46]. The proportion of preterm babies and those born with LBW or VLBW were similar for inborn and outborn infants; similar comparisons have been made in other settings [47].

Throughout the first 2 years, there was considerable variability but overall improvements in the delivery of neonatal care. This was driven in large part by the availability of antibiotics, laboratory testing, oxygen, and electricity; however, political instability, holidays, nursing schedules, and seasonal conditions also contributed to the unpredictability of unit conditions. Hot summer temperatures in a crowded unit were dangerous for infection control and hurricane-season rains often flooded the unit. Power outages were common at night and parents often slept on the floor of the unit by the neonatal beds. During renovations, the beds were moved to different parts of the pediatric ward, which affected the time it took to walk the babies over from the maternity ward. Over time, we were able to improve many of these conditions by renovating the neonatal space, providing better-quality furniture and equipment, as well as air conditioning and a steady supply of antibiotics and oxygen. However, the situation remained precarious due to the lack of adequate financial support from the health authorities. Therefore, it is notable that despite these challenges, survival improved over the first 2 years.

Another unexpected challenge was poor communication between the maternity and neonatal units. There was no formal referral system, where critical maternal information about labor and delivery would be communicated to the neonatal team. Additionally, neonatal care providers can have a broad influence in a hospital setting by ensuring that midwives and delivery nurses are properly trained in neonatal resuscitation and that parents are informed about maintaining hygiene, initiating breastfeeding, and recognizing newborn illnesses. This was sporadically done at HIC and limited by the poor communication across the two wards. We believe this was reflective of insufficient staffing across the hospital which led to a culture of siloed care within departments. Communication between doctors and nurses was also stifled and the power to ask questions or ask for materials resources was tied to seniority and tenure. Infrastructure was poor across the hospital due to a piecemeal project-by-project approach to building capacity rather than a strategic plan to improve hospital conditions. Better funded programs, such as HIV/AIDS prevention and treatment, were better staffed, equipped, and more efficient.

\section{Strengths and weaknesses}

There are some notable strengths to this work. Hospital neonatal mortality data are difficult to access in Haiti, so our study presents a unique opportunity to learn about mortality risks in this important population. We audited a whole patient database of hospital neonatal mortality and were able to document the outcomes over 2 years also allowing for an assessment of changes in mortality over time. We benefitted from the tireless effort of the pediatricians to document each data point and to alert us of any discrepancies with due notice. Similarly, we were able to discuss data quality issues and address inconsistencies in real time. While we could not do anything about data that were missing altogether, we do trust that the recorded data were of high quality.

This study also had several limitations. Although improvements in infrastructure and service-delivery capacity have been associated with improved neonatal survival in other settings [22], we could not meaningfully analyze the impact of specific improvements in the unit's infrastructure or health service delivery, including the provision of free medications, as these improvements were made against the backdrop of ongoing political instability. Similarly, we did not track changes in nurse staffing, electrical supply, and other service-delivery parameters set by the hospital that were beyond our control and that likely led to fluctuations in care. There was considerable unpredictability in the hospital's supply chain, which ultimately led us to secure therapeutics through the private sector. With less control over the supply chain, the providers could not ensure prescribed treatment for each neonate. We were not able to capture maternal risk factors, including intrapartum complications. Since the study was conducted among the newborns delivered at a public hospital, it cannot be generalized for the newborns delivered at home or in private hospitals and lower-level health centers. In addition, this study did not assess complications beyond the primary diagnosis. 


\section{Conclusions}

The potential to improve the quality of care in dedicated inpatient neonatal units in low- and middleincome countries cannot be ignored. There are many missed opportunities to provide effective interventions to neonates in health facilities. First, triage at birth to identify newborn distress must be prioritized to ensure a timely response, especially when treatment is available at the facility. Improving communication between the maternity and neonatal units, with a pediatrician attending complicated deliveries to identify neonates who are at risk of distress and illness, can improve the potential for recovery with earlier intervention. Second, considering the higher mortality among preterm babies, training for preterm care, especially for nurses, must be undertaken. Essential equipment, supplies, and medications, such as warmers, antibiotics, and an uninterrupted oxygen supply, should be made available in the very least. Culture confirmation capacity for suspected infections would improve care tremendously. And finally, Haiti must invest significant financial resources in the operation of newborn units, staffed with skilled personnel, at any hospital with a high-volume maternity ward or one that that receives referrals for complicated deliveries. With modest interventions, we were able to improve neonatal outcomes which is encouraging as we attempt to lower neonatal mortality in Haiti and beyond.

\section{Abbreviations \\ HIC: Hopital Immaculae Conception; ICD: International Classification of Diseases and Related Health Problems; LBW: Low Birthweight; NBW: Normal Birthweight; VLBW: Very-Low Birthweight.}

\begin{abstract}
Acknowledgements
Clinical staff in the maternity and pediatric wards at HIC were extremely helpful during implementation and included Dr. Jean Yves Domerçant (Medical Director); Dr. Lucien Gerard (Chief of Maternity); Dr. Edna Roberte (Chief of Pediatrics); Ms. Lucie Lafortune (Chief of Maternity Nursing); and Ms. Hyppolite (Chief of Pediatric Nursing). Dr. Jean Pape provided valuable guidance as needed. Our operational team in Haiti also provided significant on-theground assistance including Dr. Eli Francois (GHESKIO); Ms. Marie Shella Goda (GHESKIO); and Mr. Judner Mondelus (HIC). Alice Werbel and Katie Simpher were key Dartmouth advisors on maternal and neonatal health capacity building and made multiple visits to $\mathrm{HIC}$ to lead nurses' trainings. Dr. Rebecca Emeny (TDI) provided valuable reviews. Ms. Penny Wright was engaged in supporting our maternity ward communication efforts. Finally, would like to acknowledge the families who trusted us with their infants despite considerable challenges.
\end{abstract}

\section{Authors' contributions}

$A D$ analyzed the data, wrote the first draft of the paper, and completed all editing after review. MC and WJB conducted the study and collected all primary data. They actively participated in data analysis and manuscript preparation. EL actively participated in study and spent 2 months at HIC where she introduced U.S. standards of clinical care and data analysis. She actively participated in conceptualization of the study and in the process of data analysis. PJ was a critical on the ground facilitator of the study and link with the hospital administration and Ministry of Health. He actively participated and contributed to supervision of the quality of data collected and data analysis.
PW conceptualized the study, obtained funding, and made quarterly trips to the site to ensure quality, and with AD wrote the manuscript. All authors have read and approved the manuscript after each review.

\section{Funding}

Funding for this project was provided by the W.K. Kellogg Foundation and The Children's Prize. The funders did not participate in the design of the study; collection, analysis, and interpretation of data; or in writing the manuscript. The funders did receive annual reports on the progress of the project.

\section{Availability of data and materials}

The datasets used and/or analyzed during the current study are available from the corresponding author on reasonable request.

\section{Declarations}

\section{Ethics approval and consent to participate}

Approvals for the collection of these data was obtained from the Haitian Committee for Protection of Human Subjects at GHESKIO and the Institutional Review Board of Dartmouth-Hitchcock Medical Center (study number 02000017). An exemption for a minimal-risk study was secured and informed consent was not required from study subjects, since the data were extracted from patient charts after discharge or death. We paid careful attention to maintaining and preserving the anonymity of the subjects in any presentation of data. The results were made available for review by all the participating partners before submission for publication.

\section{Consent for publication}

Not applicable.

\section{Competing interests \\ Not applicable.}

\section{Author details}

${ }^{1}$ The Dartmouth Institute for Health Policy and Clinical Practice, Dartmouth College, Lebanon, NH, USA. 'Dartmouth-Hitchcock Medical Center, Lebanon, $\mathrm{NH}$, USA. ${ }^{3}$ Hopital Immaculae Conception, Les Cayes, Haiti. ${ }^{4}$ GHESKIO Centers, Port au Prince, Haiti.

Received: 9 October 2020 Accepted: 27 January 2022

Published online: 07 February 2022

\section{References}

1. Labrador R. Haiti's. Troubled Path to Development. Counc. Foreign Relat. [cited 2020 Apr 21]. Available from: https://www.cfr.org/backgrounder/ haitis-troubled-path-development

2. World Bank. Mortality rate, neonatal (per 1,000 live births) - Latin America \& Caribbean [Internet]. 2019 [cited 2021 Jul 21]. Available from: https:// data.worldbank.org/indicator/SH.DYN.NMRT?locations=ZJ

3. Global Burden of Disease Collaborative Network. Global Burden of Disease Study 2019 (GBD 2019) Results. Seattle: Institute for Health Metrics and Evaluation (IHME); 2020. Available from: http://ghdx.healthdata.org/ gbd-results-tool

4. Tura G, Fantahun M, Worku A. The effect of health facility delivery on neonatal mortality: systematic review and meta-analysis. BMC Pregnancy Childbirth. 2013;13:18.

5. ICF. The DHS Program STATcompiler. Funded by USAID. http://www.statc ompiler.com. 2015.

6. Valcin J, Jean-Charles S, Malfa A, Tucker R, Dorcélus L, Gautier J, et al. Mortality, morbidity and clinical care in a referral neonatal intensive care unit in Haiti. PLoS One. 2020;15:e0240465.

7. Boulos A, Rand K, Johnson JA, Gautier J, Koster M. Neonatal Sepsis in Haiti. J Trop Pediatr. 2017;63:70-3.

8. Dev A, Kivland C, Faustin M, Turnier O, Bell T, Leger MD. Perceptions of isolation during facility births in Haiti - a qualitative study. Reprod Health. 2019;16:185.

9. Raymondville M, Rodriguez CA, Richterman A, Jerome G, Katz A, Gilbert $\mathrm{H}$, et al. Barriers and facilitators influencing facility-based childbirth in 
rural Haiti: a mixed method study with a convergent design. BMJ Glob Health. 2020;5:e002526.

10. Lowrance DW, Tappero JW, Poncelet JL, Etienne C, Frieden TR, Delsoins D. Public Health Progress in Haiti. Am J Trop Med Hyg. 2017;97:1-3.

11. Mathon D, Apparicio P, Lachapelle U. Cross-border spatial accessibility of health care in the North-East Department of Haiti. Int J Health Geogr. 2018;17:36.

12. Ministère de la Santé Publique et de la Population. Plan Directeur de Santé (2012-2022) [Internet]. Port au Prince, Haiti: MSPP; 2013 Oct. Available from: https://mspp.gouv.ht/site/downloads/Plan\%20Directeur\% 20de\%20Sante\%202012\%202022\%20version\%20web.pdf

13. Ministère de la Santé Publique et de la Population. Oct. In: Le Paquet Essentiel de Services. Port au Prince: MSPP; 2015. Available from: https:// mspp.gouv.ht/site/downloads/Manuel\%20du\%20PES\%20Lancement\% 201 er\%20Septembre\%202016\%20compressed.pdf.

14. World Bank Group. Better Spending, Better Care: A Look at Haiti's Health Financing. 2017. Available from: https://openknowledge.worldbank.org/ bitstream/handle/10986/29517/116682.pdf?sequence=10\&isAllowed=y

15. Kemp CG, Sorensen R, Puttkammer N, Grand'Pierre R, Honoré JG, Lipira L, et al. Health facility readiness and facility-based birth in Haiti: a maximum likelihood approach to linking household and facility data. J Glob Health Rep. International Society of. Glob Health. 2018;2:e2018023.

16. Winter R, Yourkavitch J, Wang W, Mallick L. Assessment of health facility capacity to provide newborn care in Bangladesh, Haiti, Malawi, Senegal, and Tanzania. J Glob Health. 2017;7:020509.

17. Jacobs LD, Judd TM, Bhutta ZA. Addressing the Child and Maternal Mortality Crisis in Haiti through a Central Referral Hospital Providing Countrywide Care. Perm J. 2016;20:59-70.

18. Bridwell M, Handzel E, Hynes M, Jean-Louis R, Fitter D, Hogue C, et al. Hypertensive disorders in pregnancy and maternal and neonatal outcomes in Haiti: the importance of surveillance and data collection. BMC Pregnancy Childbirth BioMed Central. 2019;19:1-11.

19. Dev A, O'Hern K, Domerçant JY, Lucien G, Lucie L, Grand-Pierre R, et al. A retrospective review of facility-level obstetric complications and stillbirths in southern Haiti, 2013-2016. Rev Panam Salud Pública. 2019;43:1.

20. Raghuraman N, March MI, Hacker MR, Modest AM, Wenger J, Narcisse R, et al. Adverse maternal and fetal outcomes and deaths related to preeclampsia and eclampsia in Haiti. Pregnancy Hypertens. 2014;4:279-86.

21. Goldenberg RL, McClure EM, Saleem S. Improving pregnancy outcomes in low- and middle-income countries. Reprod Health. 2018; [cited 2019 Sep 18];15. Available from: https://www.ncbi.nlm.nih.gov/pmc/articles/ PMC6019988/.

22. Goyet S, Broch-Alvarez V, Becker C. Quality improvement in maternal and newborn healthcare: lessons from programmes supported by the German development organisation in Africa and Asia. BMJ Glob Health. 2019; [cited 2019 Nov 26];4. Available from: https://gh.bmj.com/content/4/5/ e001562.

23. Facebook Connectivity Lab, Center for International Earth Science Information Network - CIESIN - Columbia University. High Resolution Settlement Layer (HRSL). Source imagery for HRSL. DigitalGlobe; 2016 [cited 2020 Apr 16]. Available from: https://data.humdata.org/dataset/ haiti-high-resolution-population-density-maps-demographic-estimates

24. Newborn C, on F and. Levels of Neonatal Care. Pediatrics. American Academy of Pediatrics. 2004;114:1341-7.

25. Helping Babies Breathe, Second Edition: A model for strengthening educational programs to increase global newborn survival | global health: science and practice. [cited 2020 Feb 10]. Available from: https://www. ghspjournal.org/content/6/3/538.short

26. American Academy of Pediatrics. Essential Care for Every Baby.

27. WHO. ICD-10 online versions. WHO. World Health Organization; [cited 2020 Apr 16]. Available from: http://www.who.int/classifications/icd/ icdonlineversions/en/

28. Burstein R, Henry NJ, Collison ML, Marczak LB, Sligar A, Watson S, et al. Mapping 123 million neonatal, infant and child deaths between 2000 and 2017. Nature. 2019;574:353-8.

29. Wu Y. In: Nordli D, Weisman L, Dashe J, editors. Clinical features, diagnosis, and treatment of neonatal encephalopathy; 2021. UpToDate. [cited 2021 Oct 11]; Available from: https://www.uptodate.com/contents/clinicalfeatures-diagnosis-and-treatment-of-neonatal-encephalopathy?search= sarnat\%20score\&source = search_result\&selectedTitle =1 150\&usage_ type $=$ default\&display_rank $=1$.
30. Andegiorgish AK, Andemariam M, Temesghen S, Ogbai L, Ogbe Z, Zeng L. Neonatal mortality and associated factors in the specialized neonatal care unit Asmara. Eritrea BMC Public Health. 2020;20:10.

31. Roro EM, Tumtu MI, Gebre DS. Predictors, causes, and trends of neonatal mortality at Nekemte Referral Hospital, east Wollega Zone, western Ethiopia (2010-2014). Retrospective cohort study. PLoS One. 2019;14:e221513.

32. Audu LI, Otuneye AT, Mairami AB, Mukhtar-Yola M, Mshelia L. Determination of neonatal case-specific fatality rates in a tertiary health institution in North Central Nigeria. BMC Pediatr. 2021;21:302.

33. Ezechukwu CC, Ugochukwu EF, Egbuonu I, Chukwuka JO. Risk Factors For Neonatal Mortality In A Regional Tertiary Hospital In Nigeria. Niger J Clin Pract. 2004;7:50-2.

34. Desalew A, Sintayehu Y, Teferi N, Amare F, Geda B, Worku T, et al. Cause and predictors of neonatal mortality among neonates admitted to neonatal intensive care units of public hospitals in eastern Ethiopia: a facility-based prospective follow-up study. BMC Pediatr. 2020;20:160.

35. Owusu BA, Lim A, Makaje N, Wobil P, SameAe A. Neonatal mortality at the neonatal unit: the situation at a teaching hospital in Ghana. Afr Health Sci. 2018;18:369-77.

36. Adem A, Dache A, Dona A. Determinants of neonatal mortality among newborns admitted in neonatal intensive care unit at Dilla University Referral Hospital in Gedeo Zone, Southern, Ethiopia: unmatched case control study. BMC Pediatr. 2021;21:307.

37. Ndelema B, Van den Bergh R, Manzi M, van den Boogaard W, Kosgei RJ, Zuniga I, et al. Low-tech, high impact: care for premature neonates in a district hospital in Burundi. A way forward to decrease neonatal mortality. BMC Res Notes. 2016;9:28.

38. Jain K, Sankar MJ, Nangia S, Ballambattu VB, Sundaram V, Ramji S, et al. Causes of death in preterm neonates ( $<33$ weeks) born in tertiary care hospitals in India: analysis of three large prospective multicentric cohorts. J Perinatol. 2019;39:13-9.

39. Blencowe H, Cousens S, Chou D, Oestergaard M, Say L, Moller A-B, et al. Born Too Soon: The global epidemiology of 15 million preterm births. Reprod Health. 2013;10:S2.

40. Lawn JE, Gravett MG, Nunes TM, Rubens CE, Stanton C, GAPPS Review Group. Global report on preterm birth and stillbirth (1 of 7): definitions, description of the burden and opportunities to improve data. BMC Pregnancy Childbirth. 2010;10(Suppl 1):S1.

41. Liang L, Kotadia N, English L, Kissoon N, Ansermino JM, Kabakyenga J, et al. Predictors of mortality in neonates and infants hospitalized with sepsis or serious infections in developing countries: a systematic review. Front Pediatr. 2018; [cited 2021 Aug 10];0. Available from: https://internaljournal.frontiersin.org/articles/10.3389/fped.2018.00277/full.

42. Omoigberal Al, Sadoh WE, Nwaneri DU. A 4 Year Review of Neonatal Outcome at the University of Benin Teaching Hospital, Benin City. Niger J Clin Pract [Internet]. 2010; [cited 2021 Aug 10];13. Available from: http:// www.ajol.info/index.php/njcp/article/view/59787.

43. Lee AC, Mullany LC, Tielsch JM, Katz J, Khatry SK, LeClerq SC, et al. Risk factors for neonatal mortality due to birth asphyxia in southern nepal: a prospective, community-based cohort study. Pediatrics. 2008;121:e1381-90.

44. Wang W, Winner M, Burgert-Brucker CR. Limited service availability, readiness, and use of facility-based delivery care in haiti: a study linking health facility data and population data. Glob Health Sci Pract. 2017;5:244-60.

45. Ozminkowski RJ, Wortman PM, Roloff DW. Inborn/outborn status and neonatal survival: a meta-analysis of non-randomized studies. Stat Med. 1988;7:1207-21.

46. Segal. The bacterial profile of neonatal sepsis and antibiotic use in the tertiary care NICU of Kosovo. J Pediatr Neonatal Care. 2018; [cited 2020 Apr 21];Volume 8. Available from: https://medcraveonline.com/JPNC/ JPNC-08-00319.pdf.

47. Zonneveld R, Holband N, Bertolini A, Bardi F, Lissone NPA, Dijk PH, et al. Improved referral and survival of newborns after scaling up of intensive care in Suriname. BMC Pediatr. 2017;17:189.

\section{Publisher's Note}

Springer Nature remains neutral with regard to jurisdictional claims in published maps and institutional affiliations. 